\title{
The Role of Placemaking Approach in Revitalising AL-ULA Heritage Site: Linkage and Access as Key Factors
}

\author{
Ahmed M.R.M. Mohamed*, Salwa Samarghandi, Haitham Samir, Mohammed F.M. Mohammed \\ Department of Architecture, Effat University, Jeddah 21478, Kingdom of Saudi Arabia
}

Corresponding Author Email: arefaat@effatuniversity.edu.sa

https://doi.org/10.18280/ijsdp.150616

Received: 15 January 2020

Accepted: 18 July 2020

\section{Keywords:}

urban revitalization, heritage sites, placemaking, cultural tourism, users' experience, space syntax

\begin{abstract}
Tourists visiting urban heritage sites are guided by constructed itineraries that are shaped by a coherent spatial structure. This article studies the shaping of these itineraries in the heritage city of Al-Ula, using space syntax technique. The main hypothesis is that a placemaking approach of a destination based on its specificities will be able to contribute to the tourism development and place attractiveness. Building on previously developed place-making models, this study focuses on how a well-accessed and linked heritage site can be modelled and evaluated via Space Syntax measures to improve the quality of tourists' spatial experience. As the use of space syntax was found to be a useful technique for analysing tourists' itineraries and the factors that affect it; This study uses it to suggest the most appropriate pedestrian walkway given the various constraints of the existing historic fabric and to identify multiple measures of a destination's assessment. These measures have been noted in the previously developed models. The adopted measures that address access \& linkage parameters include connectivity, integration, choice, and legibility. The findings explain that the adoption of access \& linkage parameters contribute to revitalising the place in the sense of connecting the entire heritage site and enhancing the visitors experience.
\end{abstract}

\section{INTRODUCTION}

Access and linkage are as a significant feature connecting urban public spaces with buildings. The quality of any urban heritage destination is contingent on different factors, which affect how visitors navigate, move and enjoy their visit. A functional linkage in heritage sites will allow visitors and tourists to connect with the important nodes and enable people to reach and appreciate the entire place as well as the individual buildings. This paper focuses on the pedestrian linkage design in the urban heritage district of Al- Ula with the question of making comprehensive links that promote the idea of placemaking. The objective of the study is to propose a methodological approach using space syntax as a tool to investigate the needs of pedestrian linkages at the Heritage District of Al- Ula and to assess urban design factors that attract pedestrian users in the study area. The advantage of using space syntax is its capability to capture the inherited features of the settlement quantitatively and to reflect its spatial features. Thus, it offers a concrete framework not only to evaluate the existing situation but also to highlight the appropriate interventions for development.

The concept of place encompasses a wide range of attributes when it comes to heritage, as it holds memories, authentic identity, and culture. It considers being the living connection to the past, where the spirit of communities is stored. Place, therefore, is created as a response to human needs and human scale, regardless of its era. It gets develop organically from many social and cultural relationships in which it becomes a product of people who built it, their contextual environment and history [1].

\section{HERITAGE SITES AND PLACEMAKING}

The idea of placemaking is not new. However, attempts to develop a comprehensive placemaking model has been initiated by scholars recently. Project for public Spaces (PPS) is a planning, design and educational organisation proposed a placemaking model aims to achieve more prosperous places. In their work, they suggested that cities need destinations to give identity and image to their communities. Moreover, it helps to attract new residents, tourists, businesses, and investments. However, it also needs an active community destination where it can attract people. It is essential for developing a city or a region to develop destinations where people want to be. A key factor for achieving successful and thriving destinations to integrate various places [2]. Furthermore, (PPS) added that also every place should integrate multiple activities to do. In that sense, vitality, livability, and activities of a place or destinations are key aspects of what makes a significant city development. The attempted model generated by (PPS) is categorized into four main attributes that contribute to strengthening the connection between people and the places. The key attributes associated with this model are Access \& Linkages, Comfort \& Image, Uses \& Activities, and Sociability [2], Figure 1.

In her study [3], (Salwa) developed this model to be applicable on Heritage sites considering the human aspect that ensures a pleasant experience for all visitors and users of heritage sites. She added two main attributes to the (PPS) original model. These domains are named as, "context and conservation" and "site interpretation". The former is concerned with the physical setting of the heritage site that 
consists of preserved, protected, valuable, aesthetics and urban context integration. While the later refers to the intangible aspects of the heritage itself incorporating memorable, soulful, authentic, informative and human-oriented experience, Figure 2.

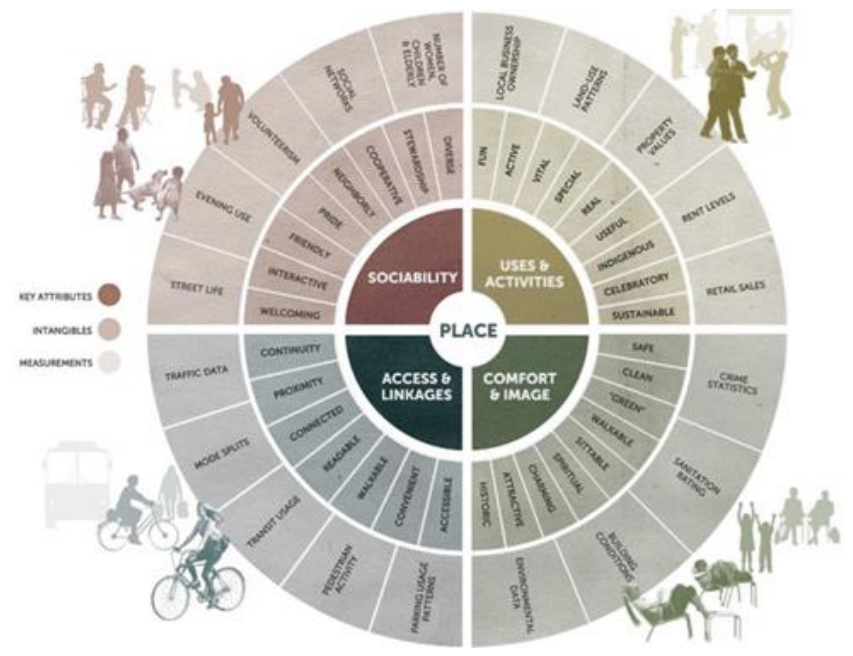

Figure 1. PPS placemaking model [2]

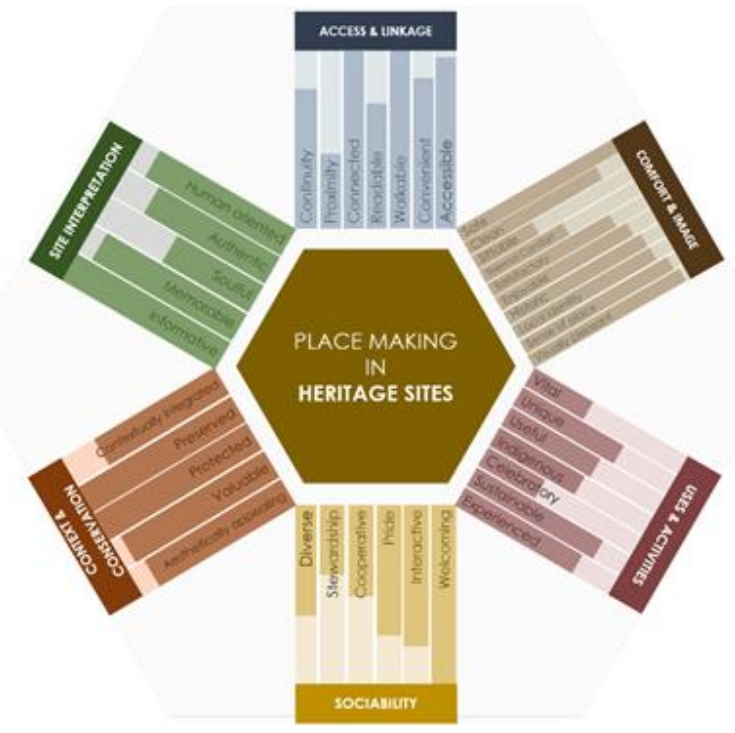

Figure 2. Placemaking model for heritage sites [3]

\section{THE ROLE OF ACCESS AND LINKAGE IN PLACEMAKING OF HERITAGE SITES}

This paper focuses on the physical interventions that promote the revitalization of Al-Ula old town. Therefore, the access and linkage attribute were selected due to its strong connection to the physical context.

One can easily judge a place by its accessibility and its connection to the surroundings. A key success for any place is that can be easily reached, entered and smoothly navigated. It must be arranged so people can access to most of what is going through the place either in distance or in close. Another important element, which can make it accessible, is the edges of its public places. In heritage sites, aligning historic structures and featured buildings with coherent networks creates vibrancy and attractiveness for people to walk, and explore.

\subsection{The essence of a place \& cultural tourism}

Sense of place is considered a pivotal concept in successful urban design where it belongs to an invisible meaning and attachment between man and place [4]. Sense of place has been defined in many ways; however, this study agreed on defining the sense of place according to (Wales tourist board) that it is the feeling you perceive when you reach a new place for the first time which can be a thousand and one thing. It is about the first sight, look, feeling the environment, the context, and the people, sound, which are inherited in the place. While another definition by (Steve Thorne, cultural tourism specialist) has defined sense of place as what person directly senses from a distinctly a new visited place out of his context, which can be called an ambience of a place, mixture of landscapes which are expressed in a way that mesmerise one's emotions [5]. This ambience can only be achieved by creating an accessible, connected, and linked context.

Therefore, it is clear that placemaking approach (access \& Linkage attribute) can contribute to achieving successful cultural tourism in heritage places, where it considers achieving a humanized cultural tourism for an ultimate and unique experience. In that sense, establishing an integrated model that combines continuity, connected, readable, and accessible considerations for revitalisation is significant. Ensuring to provide a rich and authentic experience for both visitors and tourists; where they could take back home a collection of feelings, knowledge and physical elements such as souvenirs of history [6].

\subsection{Visitors' experience \& cultural tourism}

Visitors' experience plays a critical role in the conceptualisation of heritage tourism. In his study Apostolakis, argues that heritage tourism industry had shifted from a product-focused orientation toward a customer-focused orientation [7]. Visitors are considered as the customers, and their needs must be responded accordingly to maximize satisfaction and loyalty. The marketing concept emphasizes on satisfying customers' needs, through the integrated efforts of all functional elements to enhance the organization's performance [8]. This approach is one of the most important methods that can effectively affect the performance of cultural heritage destinations [9]. Thereby, attention to heritage and cultural places will increase regarding the use of those visitors. Due to this, the need to develop and protect the heritage places are essential to maintain them accordingly. Besides, to prepare those places to host people for visits and walking, by adding supportive services and required needs of tourists [10].

It is essential to prepare the site with a good network of pedestrian walkways given the existing urban fabric, open spaces, the significance of heritage buildings from one side and the visitors' needs and requirements. To be followed by simulating the visitor's/tourists feelings by ensuring the welcoming level and their visual impression of the place, how easy was reaching the destination, accessibility level and provided services adequately among the place. Moreover, it is essential to provide information centers among the heritage places, in which it serves people at all times. These centers should be also located in visible and attractive locations, where also it should provide the needed signs, maps, tours offer, and many others to guide people [11].

The places should be also supported by range of services like parking areas, public sanitation, places to eat and chill, 
markets, and others by providing convenient open spaces. Another important factor is to provide a readable context of the heritage and cultural places, where it helps the tourist and visitors to move around the place and provide them with needed information. Finally, preparing the activities that can make the place livable, memorable and enjoyable. In this sense, it is important to allocate the locations of these elements in a methodological way that improves the visitor experience.

\section{CASE STUDY}

This paper will focus on evaluating the proposed development of one of Al-Ula projects which is the revitalization of Al-Ula old-town [12].

\subsection{Al-Ula heritage site}

Al Ula town is located in the north-western region of the kingdom of Saudi Arabia in between Al-Madinah AlMunawara and Tabuk Figure 3. The extraordinary features of Al-Ula natural environment have created a unique destination for various civilisations to establish and flourish. Such as, Dedanites, Nabataeans, Lihyanites who built their empires among the city boarders and left lots of their imprints on surrounding mountains. Al-Ula got its importance as it used to be the main crossroad for traders routs between the various civilisation of India, Egypt, Syria and Iraq. This has exposed Al-Ula to become a place for cultural and trading exchange throughout history. Al-Heger area which was civilised by AlNabatean commodity which also known as Mada'en Saleh, the magnificent ancient city and the first registered UNESCO site in Saudi Arabia. Its unique, remarkable mountains façade engravings make it an important historical place. In addition to Mada'en Saleh, Al-Ula was hometown to other vital civilisations that left several of substantial historical and archaeological sites such as Al-Dadan and Lihyanees kingdoms. Moreover, Al-Hijaz railways stations and the significant Ekmaa mountain, which was considered an open library where there was found over hundreds of early Arabic inscriptions going back to Dadan and Lihyanite periods [13].

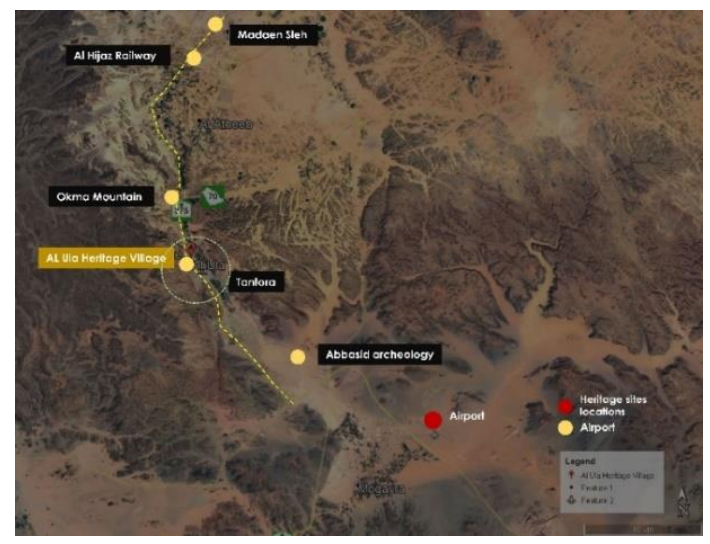

Figure 3. Al-Ula old-town [3]

Al-Ula old-town is considered as one of the important three heritage Islamic cities worldwide, where this town resembles the authentic Islamic old town by its unique houses structures, mosques and markets. Where also its unique strategic location across the main road and in the heart of Al-Ula and overlooking a vast farm where they used to work surrounded by a massive mountain in the back, Figure 4. Al-Ula old-town has three significant landmarks, which give it more value as it has Moussa Bin Nassier Castel in upfront of the city overlooking the entire village and the main road, Figure 3. Also, it has the oldest mosque in the Islamic empire, which is called the Rock mosque where Prophet Mohammed, peace be upon him, prayed inside it. In addition to the famous Tantoura yard, where people have built a sign on understanding timings and seasons of the year as it works like sun clock [14].

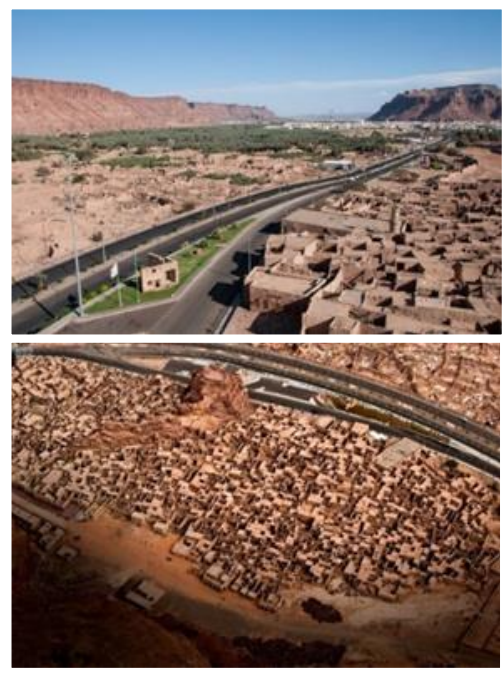

Figure 4. Al-Ula old-town, showing the archaeological site [3]

The old town is featured by its unique architecture and urban fabric, where houses were built depending on each other and mainly have one to two floors only. It was designed according to their needs, as the ground floor is primarily open space for welcoming people and work and the first floor for living. Crossing those clay structures a tiny allies where there two primary spines which passing across the entire village. The main materials used in buildings are traditional such as clay, palm fronds and wood, Figure 5. Al-Ula residents occupied Al Ula old town for years until they all left it for 70 years until it became abandoned. The city remained abandoned for years where it was deteriorated and many parts of it were damaged because of natural factors like rain as well as the neglection of authorities [14].

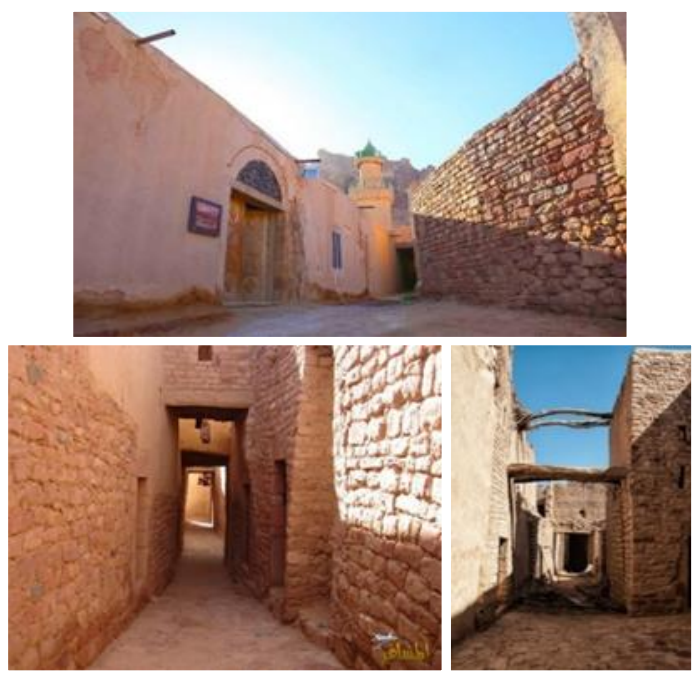

Figure 5. Architectural features of Al-Ula old-town [3] 
Aligning with 2030 vision of Saudi Arabia, the government took many initiatives to revitalise Al-Ula area. First, it established a dedicated governmental organisation for Al Ula development, which is the Royal Commission of Al-Ula (RCU). The goal of this organisation is to preserve historical and heritage sites among Al-Ula as well as documenting the historic features by a team of an international and local archaeologist to discover the area intensely. The aim is developing the city to become a developed touristic destination where it could host visitors from all over the world [12].

\subsection{Case study analysis}

Al-Ula old-town, showing the archaeological site [3], is confronted with the challenge of converting its historically valuable urban fabric that is no longer used for its original purpose, and which represents an opportunity to bring in touristic uses. The old buildings can potentially be used for many kinds of activities. However, architecturally appealing they may be, the size and scale of these abandoned buildings organised in organic patterns make it difficult to find new uses for them. Looking merely to the utilisation of the old buildings without considering how visitors of the site would experience them and move in between different destination will result in a limited vision. The small alleyways, as well as the spaces between buildings, should be carefully designed to ensure the best experience for the visitors and the best exposure to the heritage buildings which still stands as a manifestation of the city cultural and historic patrimony.

This research aims to restore liveability of Al-Ula old-town via the development of its urban context. In this sense, the authors adopted the placemaking approach focusing on access and linkage domain Figure 6. This domain includes seven attributes with different importance: connected and walkable have of high significance, while accessibility, convenient, readable, and continuity have medium importance, whereas proximity has of low significance [6]. To achieve these attributes and fulfil the access and linkage domain, 10 parameters should be accomplished. Because of the historical nature of the site, minimum interventions can be applied to maintain its authentic identity and character.

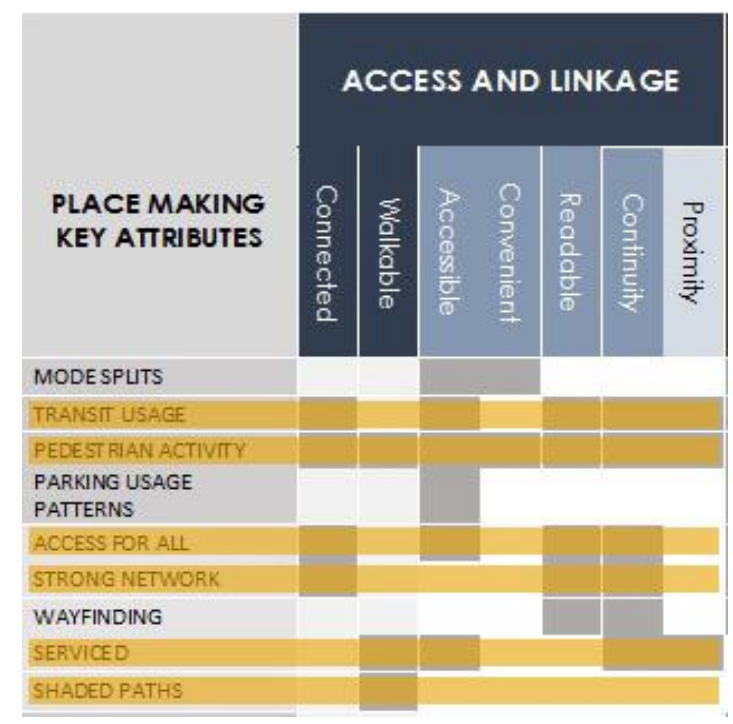

Figure 6. Key attributes of access and linkage domain
Consequently, the revitalisation proposal focused mainly on the parameters addressing the high importance attributes and/or a wide number of these attributes. In this sense, the proposal focused on six parameters: pedestrian activity, transit usage, services, access for all, strong network and shaded paths Figure 6. Through the analysis of the urban context of Al-Ula old-town, some parameters can be achieved smoothly without a massive disturbance. For example, some buildings can be reused to provide services and offering pedestrian activities such as cafes and restaurants. Also, the compact pattern offers shaded paths for all inner streets which represent the focus of the development.

However, to achieve other parameters - transit usage, access for all and strong network - there was a need to change the physical elements of Al-Ula old-town urban context, without compromising its identity and character. The parameters described above addressing mainly the spatial structure of the site which needs to be well connected and more readable. In real, enhancing connectivity and legibility is not only addressing these three parameters but also encouraging pedestrian activity and walkability. To achieve this aim the development proposal attempts to strengthen connectivity and legibility of the spatial structure via demolishing weak and damaged buildings in the site. However, the selection of these buildings had been made with a high degree of sensitivity to maintain the identity of the site. Also, the proposal focused on creating a main internal path that links the whole site longitudinally as the whole transversal inner paths emphasis on dividing the site into segregated zones, Figure 7.

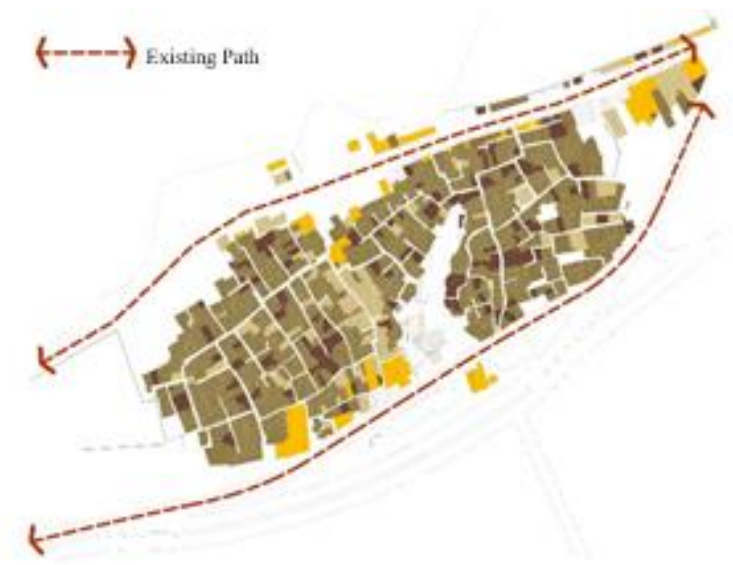

(a)

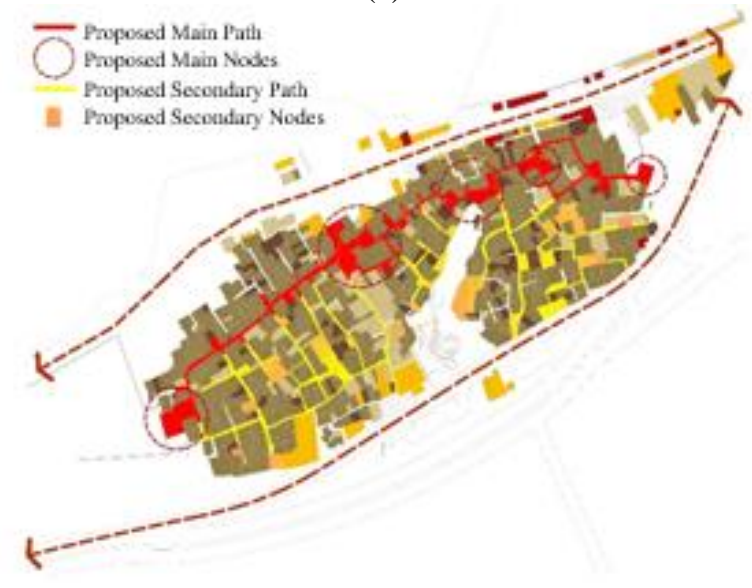

(b)

Figure 7. Al-Ula old-town. (a) current situation; (b) proposed development 
Additionally; the new longitudinal path offers more comfortable and enriches walkable experience of the historical site rather than the poor experience offered by the existing peripheral paths. However, there is a need to evaluate the proposed development and test its validity. In this regard, and because the focus was on the spatial structure of the site, the research adopted space syntax methodology, Figure 5.

\subsubsection{Methodology}

To evaluate the proposed development of Al-Ula old-town, the present research adopted space syntax methodology. The advantage of this methodology is the ability to measure and compare quantitatively the urban context features such as connectivity, accessibility and legibility [8] \& [9]. In this sense; it will reflect to what extent the development proposal fulfilled the access and linkage attributes and consequently enhanced the urban context of Al-Ula old-town. The space syntax measures of connectivity and integration reflect the connected and strong network parameters, while choice measure demonstrates the degree of accessibility (access for all), and measure of intelligibility reflects the legibility degree of the urban context which address the transit usage parameter, readable and way-finding attributes. Most importantly, these measures come in a quantitative way that helps in comparing between the existing situation and the proposed development to measure the degree of improvement. Also, the integration measure reflects the "to movement' type which indicates the most important destinations in the site while choice reflects the "through movement" nature which shows the most accessible and busy pass-by streets. Thus, this can contribute significantly to propose the location of important destinations and highlight the venues of the pedestrian activity and services across the historical site.

\subsubsection{Analysis}

Figure 8 shows the comparison between the axial maps of the current situation and the proposed development of Al-Ula old-town. The maps reflect the significant appearance of the proposed main path that connects the whole site from north to south, offering a new internal rich and continuity experience for pedestrian movement. This is more evident in accessibility map showing how this path becomes more accessible and attractive to "through-movement" of pedestrian and open new choices to navigate the internal structure of the site and allocate the venues of pedestrian activities and services. Regarding the integration maps, the proposed main path acquired relative importance in some segments (highlighted with red circles) suggesting new settings as important destinations within the internal context of the site which can enrich the pedestrian experience and creating magnetic spots. Regarding the connectivity maps, it shows how the proposed main path succeeded in achieving continuity across the internal street network.

At a numerical level; as shown in Table 1, the development proposal enhanced the degree of connectivity by $13.6 \%$, the global integration by $27.8 \%$ and the local integration by $11.7 \%$.
Despite the relatively minor enhancement in quantitative measures due to the constraints of maintaining the site identity, the amount of open areas and freedom given to the users to access, experience, and navigate the site has been increased significantly. This is evident in the intelligibility measure, which enhanced by $52.1 \%$ reflecting a more readable and walkable context.

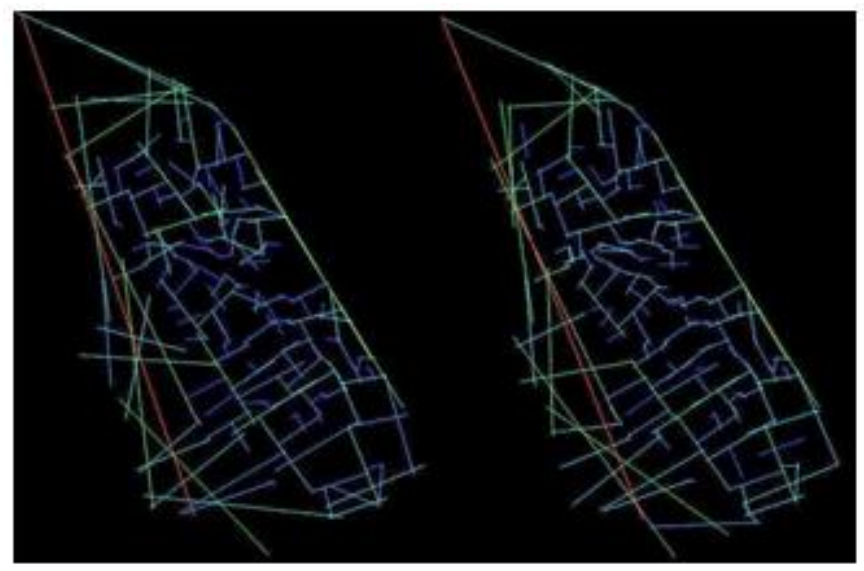

(a) Connectivity maps

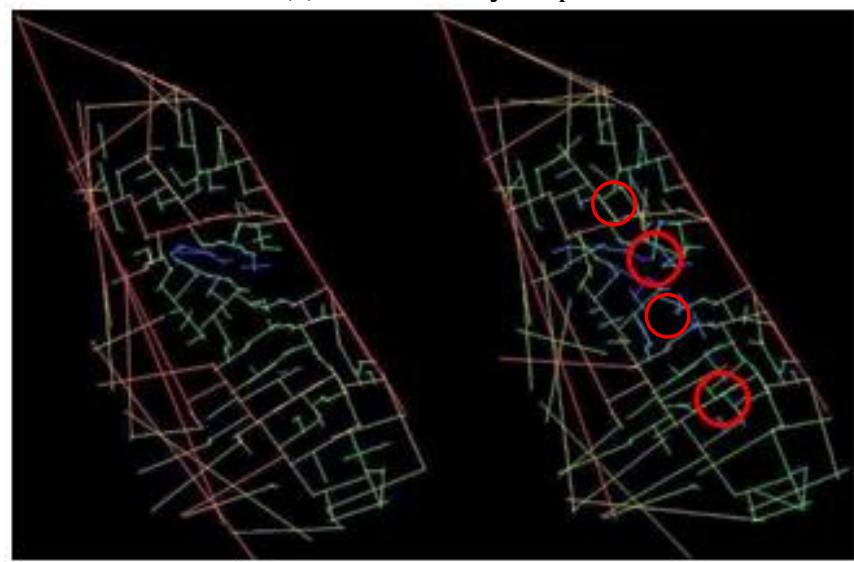

(b) Global Integration maps

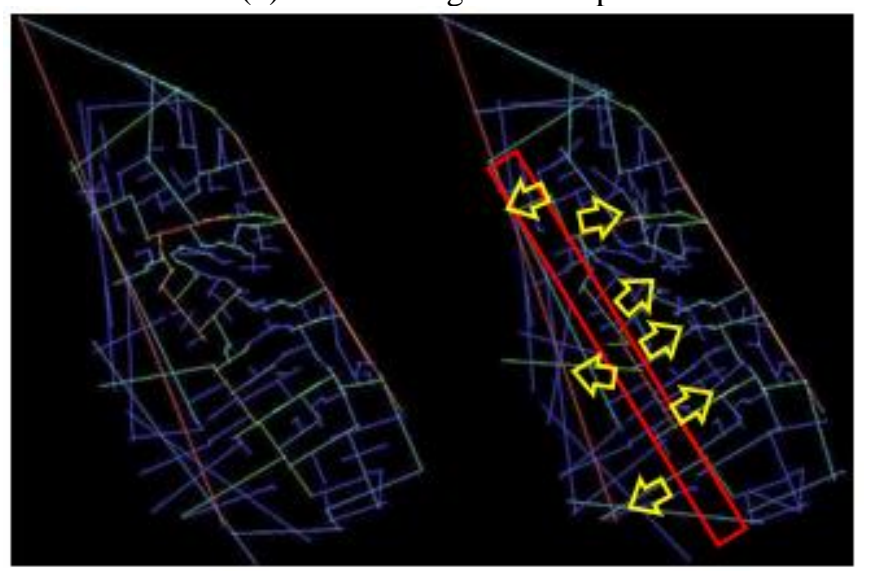

(c) Choice maps (Accessibility)

Figure 8. Axial analysis maps of Al-Ula old-town (left: current situation - right: proposed development)

Table 1. Syntactic measures of Al-Ula old-town (current vs Development proposal)

\begin{tabular}{ccccc}
\hline & Connectivity & Integration [HH] & Integration [HH] R3 & Intelligibility \\
\hline Current situation & 3.540983 & 0.89659 & 1.57173 & 0.3524 \\
Development proposal & 4.02272 & 1.14590 & 1.75031 & 0.5359 \\
\% of improvement & $13.6 \%$ & $27.8 \%$ & $11.4 \%$ & $52.1 \%$ \\
\hline
\end{tabular}




\section{CONCLUDING REMARKS}

Placemaking creates a strong bond between the physical components of the site and the visitor's experience. Focusing on visitors experience and pedestrian movement can yield significant solutions while maintaining the identity of the historical patterns, which were depending on walking as the main movement type. By investigating the placemaking models discussed in this research, it was found that access and linkage to and within the heritage site with minimum interventions play an important role in shaping the spatial experience of the tourists.

The research showed the capability of space syntax techniques to analyse the physical context of historical sites and capture their features and deficiencies, besides, to evaluate their development proposal objectively. The analysis indicated that the proposed tourist's itinerary developed by the current revitalisation plan is not the best in terms of its connectivity, integration and legibility parameters. The research, based on these measures, proposed another itinerary that has been proved to be more effective in enhancing the experience of the visitors and tourists and help in allocating the added supporting services and open spaces.

The research used the space syntax to test and compare between the current situation and the proposed development of Al-Ula old-town. This informed and validated many interventions that can add to the significant appearance of the old city and offer an internal rich and continuous experience for tourists and visitors. Two main interventions have been elaborated: the location of the proposed main path that connects the whole site from north to south, and the important segments that can be used to accommodate featured services and facilities along this path. In regard to quantitative measures, it was noticed the minor enhancement of the development on the degree of connectivity, global integration, and local integration. Whereas, the amount of open areas and freedom given to the users to access, experience, and navigate the site has been increased significantly.

Finally, space syntax analysis showed that the measures of connectivity, integration and choice (accessibility) represent the most important parameters that can be used to address a successful revitalization approach and process.

\section{REFERENCES}

[1] Hosking, N. (2019). Placemaking: The Heart of Heritage Conservation. (M. Strebe, Ed.). https://globalheritagefund.org/, accessed on Dec. 24, 2019.
[2] PPS. Placemaking, What if we Build Cities around Places? Project for Public Spaces Inc. https://www.pps.org/, accessed on Dec. 24, 2019.

[3] Samargandi, S. (2019). Integral placemaking in sensitive heritage sites for successful cultural tourism. M.Sc. thesis of urban design. Effat University, Jeddah, KSA. https://www.researchgate.net/publication/335128725_In tegral_Placemaking_in_Sensitive_Heritage_Sites_for_s uccessful_cultural tourism.

[4] Salah Ouf, A.M. (2001). Authenticity and the sense of place in urban design. Journal of Urban Design, 6(1): 7386. https://doi.org/10.1080/13574800120032914

[5] Strong, M.J. (2004). Making sense of place: A formative case study of Canada. M. Thesis in city planning, University of Manitoba, Canada. https://mspace.lib.umanitoba.ca/xmlui/handle/1993/157 44.

[6] Hosagrahar, J., Pascual, J. (2016). Why must culture be at the heart of sustainable urban development. Culture, 21,36 , https://www.agenda21 culture.net, accessed on Nov. 15, 2018.

[7] Haitham, S., Salwa, S., Mohammed, F.M.M. (2019). Placemaking as an Approach to Foster Cultural Tourism in Heritage Sites. WIT Transactions on the Built Environment, 191: 321-338. https://doi.org/10.2495/STR190281

[8] Hillier, B., Hanson, J. (1984). The Social Logic of Space. Cambridge University Press. https://doi.org/10.1017/CBO9780511597237

[9] Refaat, A.M. (2019). Space Syntax Theory and Its Contribution to Urban Design. In H. Abusaada, C. Vellguth, \& A. Elshater, Handbook of Research on Digital Research Methods and Architectural Tools in Urban Planning and Design, Hershey, Pennsylvania: IGI Global, 203-222. https://doi.org/10.4018/978-1-52259238-9.ch010

[10] Ghadeer, A., Hatoon J., Haitham, S. (2018). Enhancing Cultural Tourism Experience: The Case of Historical old Jeddah. WIT Transactions on the Built Environment, 177: 39-50. https://doi.org/10.2495/IHA180041

[11] Spoon, S.C. (2005). What defines walkability: Walking behavior correlates. Master Project, the University of Northern Carolina, USA.

[12] Saudi Commission for Tourism and Antiques (2016). AlUla Old Town Revitalization Project Studies, KSA.

[13] Royal commission of AlUla (2018). About AlUla, https://www.rcu.gov.sa/en/about-us/about-alula, accessed on Nov. 22, 2019.

[14] Cultural heritage department, Saudi Commission for Tourism and Antiques (2016). Architectural Documentation of AlUla Old Town, KSA. 